\title{
Image Denoising Using New Proposed Method Based on Wavelet Transform for Different Wavelet Families
}

\author{
Pushpa Koranga ${ }^{1}$, Garima Singh ${ }^{2}$, Dikendra Verma ${ }^{3}$ \\ ${ }^{1}$ Department of Electronics and Communication engineering, GEHU \\ Bhimtal, Uttarakhand, India \\ Pushpakoranga14@gmail.com \\ ${ }^{2}$ Department of Electronics and Communication engineering, GEHU \\ Bhimtal, Uttarakhand, India \\ Singh.garima993@gmail.com \\ ${ }^{3}$ Department of Electronics and Communication engineering, GEHU \\ Bhimtal, Uttarakhand, India \\ dikendraverma@gmail.com
}

\begin{abstract}
Image usually gets distorted during acquisition, processing and transition. Now a day, Wavelet transform method is getting popular for image denoising. As wavelet transform has many advantages over other method such as best localization and multiresolution properties. Wavelet transform used various techniques for image denoising such as Visu shrink but this technique have disadvantage that it produce over smoothening of image which causes blur in the edges. So to overcome such problem we have proposed new method by modifying the Visu shrink thresholding techniques. We have compared our proposed method with the Visu thresholding technique on the basis of PSNR value for different wavelet families such as Haar, Daubechies, Biorthogonal, Symlet and Coiflet.
\end{abstract}

Keyword-Visu shrink, Haar, Daubechies, Biorthogonal, Symlet, Coiflet, PSNR

\section{INTRODUCTION}

Image denoising is done to improve the quality of image by suppressing the noise level. Several method have been adopted till now for image denoising such as discrete cosine transform, filter method, fourier method etc but wavelet transform is the best method. Wavelet transform is basically non linear transformation method. Wavelet transform have best localization properties which become important tool for image denoising [1]. Wavelet has multi resolution property and in time frequency characteristics it shows good performance [2]. The main two thresholding techniques based on wavelet transform are Soft thresholding and hard thresholding. A wavelet transform are classified into two type firstly continuous wavelet transform and second discrete wavelet transform [3]. The wavelet thresholding method is effective for energy compaction [4], [5]. Wavelet transform have mainly three steps first of all wavelet transform method is applied to the input noisy image. Secondly thresholding technique is applied to the wavelet coefficients. Finally inverse wavelet transform is applied to obtain the reconstructed image [4]. In Hard or soft thresholding techniques the coefficients of wavelet transform is obtain which compared with threshold value if it is less than threshold value obtain by different method such as global threshold, Visu threshold, Bayes threshold etc then it is set to zero otherwise kept as it is or absolute value is kept [6],[7]. In mathematical form wavelet transform method can be represented as [8]-

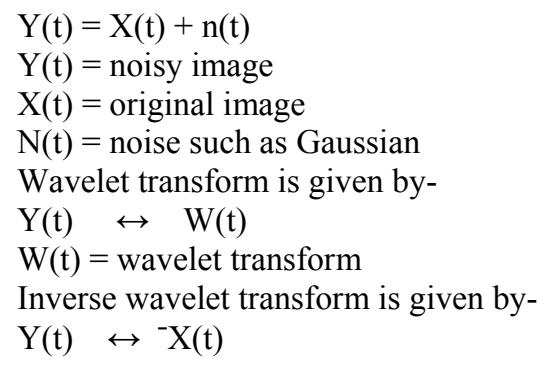


${ }^{-} \mathrm{X}(\mathrm{t})=$ inverse wavelet transform

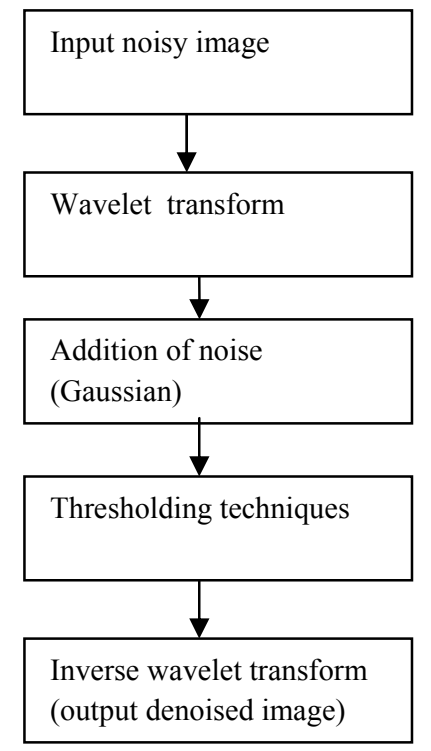

Fig. 1. Steps for Wavelet transform for Image denoising

\section{THRESHOLDING TECHNIQUES}

Universal threshold- It is the oldest method proposed by Donho and Johnstone for removing noises [6]. This method is found to be efficient for all decomposition level [9].The global threshold value is given by-

$\mathrm{T}=\boldsymbol{\sigma} \sqrt{\log m}$

$\boldsymbol{\sigma}=$ noise variance

$\mathrm{m}=$ total number of pixel in image

In this $\sigma$ is the variance which is used for wavelet shrinkage and set all detail coefficients to zero when total number of pixel leads to infinite [7]

Visu threshold- Visu shrink is thresholding techniques obtained by using the global threshold which was proposed by Donho and Johnstone. This technique is also called global threshold [7].

Visu threshold value is given by -

$\mathrm{T}=\boldsymbol{\sigma} \sqrt{2 \log m}$,

$\boldsymbol{\sigma}=$ noise variance

$\mathrm{m}=$ total number of pixel in image

\section{WAVELET FAMILIES}

A. Haar: Haar is the simplest of wavelet family. Haar wavelet is related to mathematical operation in discrete form which is called as Haar transform [10]. It is invented by Hungarian mathematician in 1910. It is discontinuous in nature and similar to step function [11]. Haar wavelet can be represented in term of orthonormal function [12]. Haar transform basically distribute discrete signal into two sub signal which is half of the original length [10].

B. Daubechies: Daubechies wavelet is discovered by the Ingrid Daubechies which can be written as dbN where $\mathrm{Db}$ represent Daubechies and $\mathrm{N}$ represent order [13]. Similar to Haar wavelet, Daubechies also distribute discrete signal into two equal sub signals and then find out difference and average of it. Daubechies is continuous in nature and has balanced frequency response. Daubechies has non linear phase response [10]. It is orthonormal which lead to high energy compaction [13].

C. Biorthogonal: Biorthogonal is symmetric in nature [12]. It is invertible but may or may not be orthogonal in nature. It takes input signal at frequency $f$ and calculates highpass and lowpass coefficients at half of the frequency f. A quadrature mirror is produce by computing highpass and lowpass coefficients in multiple stages. It has linear phase property [13].It can be written in bior form. In this paper we have used bior1.5. 
D. Symlet: Symlet is orthogonal in nature and also known as least asymmetric. It can be written in SymN form where $\mathrm{N}$ is any positive integer. Symlet wavelet family has wavelet function and scaling function where scaling function has $\mathrm{N}$ vanishing moment. It has total length of $2 \mathrm{~N}$ for compact support. It is best suited for discrete wavelet transform [12].In this paper we have used sym1.

E. Coiflet: Coiflet can also be written in coifN where $\mathrm{N}$ represents vanishing moment. It also has total number of filter tap which is $2 \mathrm{~N}[12]$. In this paper we have used Coif1.

\section{PROPOSED METHOD}

An adaptive thresholding method is proposed by modifying the Visu Shrink thresholding techniques. This new modified technique depends on the decomposition level such as LL, HL, LH and HH. Where L represents low frequency signal and $\mathrm{H}$ represent high frequency signal. One of the main disadvantages of Visu shrink is that for higher decomposition level it produces over smoothening of the image in which the edges of the image is blur in visual appearance. The main reason behind it is that with increase in decomposition level, the threshold value also increase which at some point leads to the over smoothening of the image. In our proposed method the threshold value depends on the decomposition level such that with increase in decomposition level the threshold value is maintain.

$$
\begin{aligned}
& \mathrm{T}=\sqrt{2 \log m}\left(1+\frac{1}{2^{\wedge} K}\right) \\
& \boldsymbol{\sigma}=\text { noise variance } \\
& \mathrm{K}=\text { decomposition level } \\
& \mathrm{m}=\text { total number of pixel in image }
\end{aligned}
$$

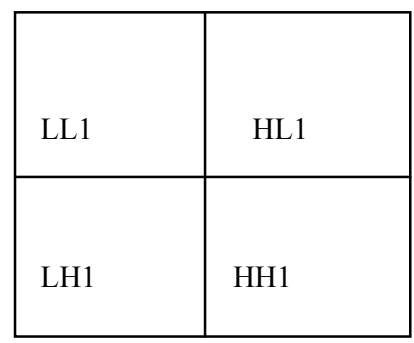

Fig. 2. Decomposition for first level

\begin{tabular}{|l|l|l|}
\hline LL2 & HL2 & \\
\cline { 1 - 1 } LH2 & HH2 & \multirow{2}{*}{ HL1 } \\
\hline LH1 & & HH1 \\
\hline
\end{tabular}

Fig. 3. Decomposition for second level

\section{EXPERIMENT AND RESULT}

In this paper we have compared Visu shrink thresholding techniques and our proposed method on the basis of PSNR value.

PSNR-PSNR stands for peak signal to noise ratio. In this image quality is measured in term of PSNR value, better the PSNR value it means better is the image quality. The image quality is given by-

$\mathrm{PSNR}=10 \log (255 / \mathrm{MSE})^{2}$

$\mathrm{MSE}=$ mean square error 
TABLE I

For noise level $\boldsymbol{\sigma}=10$ PSNR value for Visu shrink and proposed method using Gaussian noise of 2 level of decomposition

\begin{tabular}{|l|l|l|}
\hline $\begin{array}{l}\text { Wavelet } \\
\text { families }\end{array}$ & $\begin{array}{l}\text { PSNR } \\
\text { (VIsu) }\end{array}$ & $\begin{array}{l}\text { PSNR } \\
\text { (proposed) }\end{array}$ \\
\hline Haar & 24.8719 & 25.8240 \\
\hline Db1 & 24.8888 & 25.8497 \\
\hline Bior1.5 & 24.5558 & 25.4266 \\
\hline Sym1 & 24.8776 & 25.8533 \\
\hline Coif1 & 25.0344 & 26.0905 \\
\hline
\end{tabular}
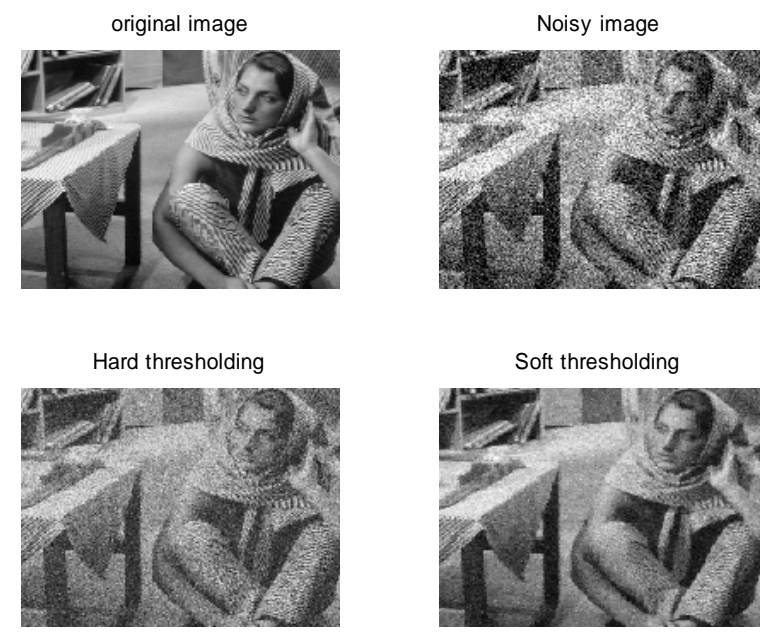

Fig. 4.For noise level $\boldsymbol{\sigma}=10$ for Haar using Visu shrink method for 2 level of decomposition
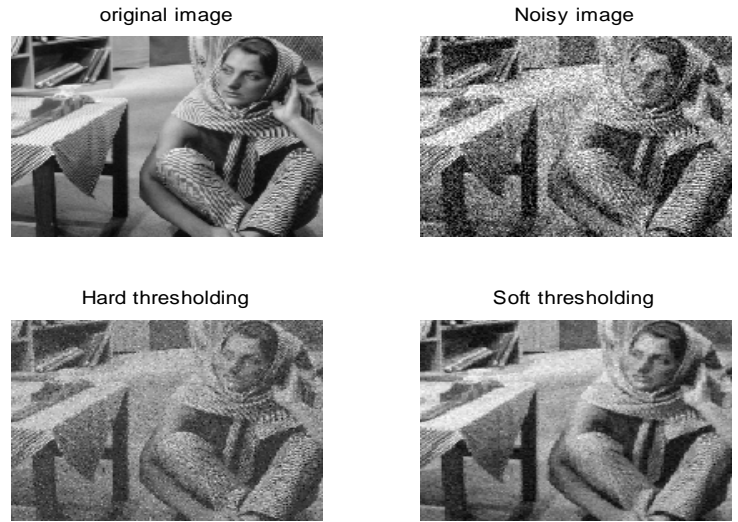

Fig. 5.For noise level $\boldsymbol{\sigma}=10$ for Haar using proposed method for 2 level of decomposition 
ISSN (Print) : 2319-8613

TABLE II

For noise level $\boldsymbol{\sigma}=15$ PSNR value for Visu shrink and proposed method using Gaussian noise of 2 level of decomposition

\begin{tabular}{|l|l|l|}
\hline $\begin{array}{l}\text { Wavelet } \\
\text { families }\end{array}$ & $\begin{array}{l}\text { PSNR } \\
\text { (VIsu) }\end{array}$ & $\begin{array}{l}\text { PSNR } \\
\text { (proposed) }\end{array}$ \\
\hline Haar & 26.8150 & 27.5406 \\
\hline Db1 & 26.8021 & 27.8341 \\
\hline Bior1.5 & 26.3197 & 27.3840 \\
\hline Sym1 & 26.8205 & 27.8627 \\
\hline Coif1 & 27.1816 & 28.3498 \\
\hline
\end{tabular}
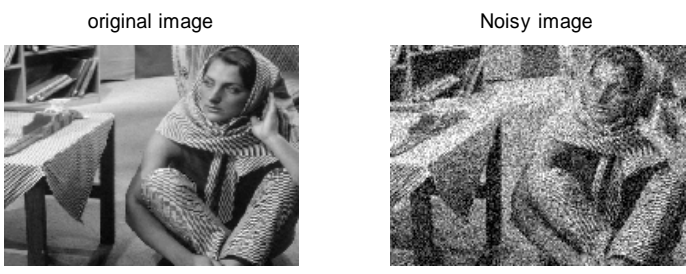

Hard thresholding

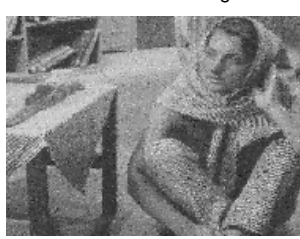

Soft thresholding

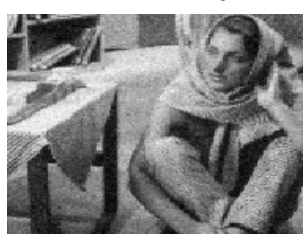

Fig. 6.For noise level $\boldsymbol{\sigma}=15$ for $\mathrm{db} 1 \mathrm{using}$ Visu shrink method for 2 level of decomposition

original image

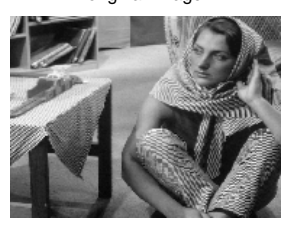

Hard thresholding

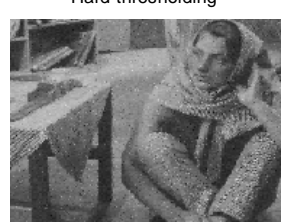

Noisy image

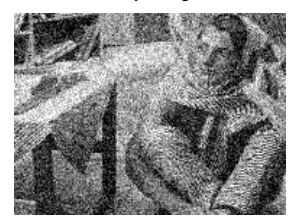

Soft thresholding

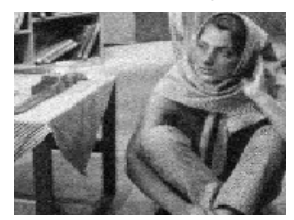

Fig. 7.For noise level $\boldsymbol{\sigma}=15$ for $\mathrm{db} 1 \mathrm{using}$ proposed method for 2 level of decomposition 
ISSN (Print) : 2319-8613

TABLE III

For noise level $\boldsymbol{\sigma}=10$ PSNR value for Visu shrink and proposed method using Gaussian noise of 4 level of decomposition

\begin{tabular}{|c|c|c|}
\hline $\begin{array}{c}\text { Wavelet } \\
\text { families }\end{array}$ & $\begin{array}{c}\text { PSNR } \\
\text { (VIsu) }\end{array}$ & $\begin{array}{c}\text { PSNR } \\
\text { (proposed) }\end{array}$ \\
\hline Haar & 24.8804 & 25.2053 \\
\hline Db1 & 24.9215 & 25.1487 \\
\hline Bior1.5 & 24.6087 & 24.7824 \\
\hline Sym1 & 24.9252 & 25.2166 \\
\hline Coif1 & 25.0878 & 25.3634 \\
\hline
\end{tabular}
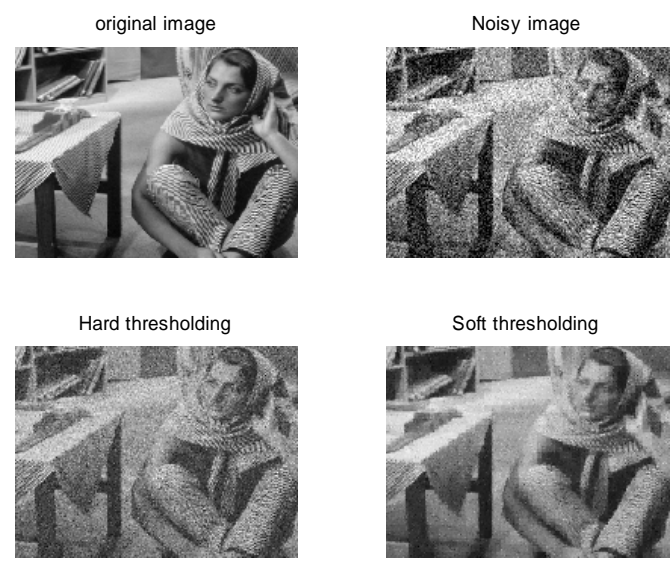

Fig. 8.For noise level $\boldsymbol{\sigma}=10$ for Haar using Visu shrink method for 4 level of decomposition
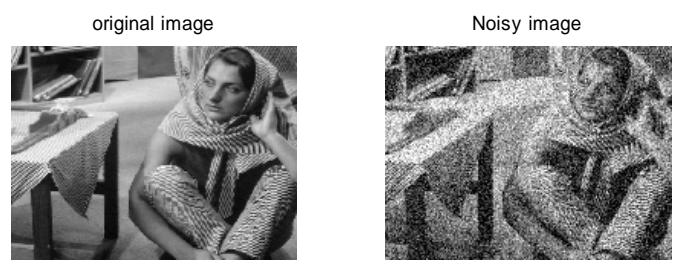

Hard thresholding

Soft thresholding
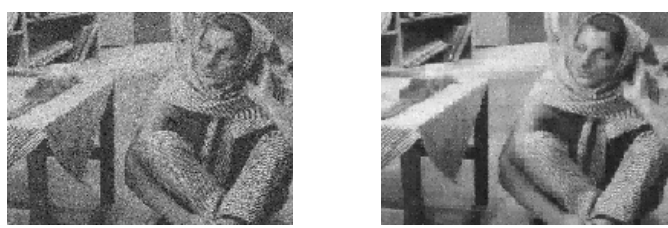

Fig. 9.For noise level $\boldsymbol{\sigma}=10$ for Haar using proposed method for 4 level of decomposition 
TABLE IV

For noise level $\boldsymbol{\sigma}=15$ PSNR value for Visu shrink and proposed method using Gaussian noise of 4 level of decomposition

\begin{tabular}{|l|l|l|}
\hline $\begin{array}{l}\text { Wavelet } \\
\text { families }\end{array}$ & $\begin{array}{l}\text { PSNR } \\
\text { (VIsu) }\end{array}$ & $\begin{array}{l}\text { PSNR } \\
\text { (proposed) }\end{array}$ \\
\hline Haar & 26.8466 & 27.1815 \\
\hline Db1 & 26.8265 & 27.1716 \\
\hline Bior1.5 & 26.3652 & 26.6821 \\
\hline Sym1 & 26.8621 & 27.1353 \\
\hline Coif1 & 27.2633 & 27.6315 \\
\hline
\end{tabular}
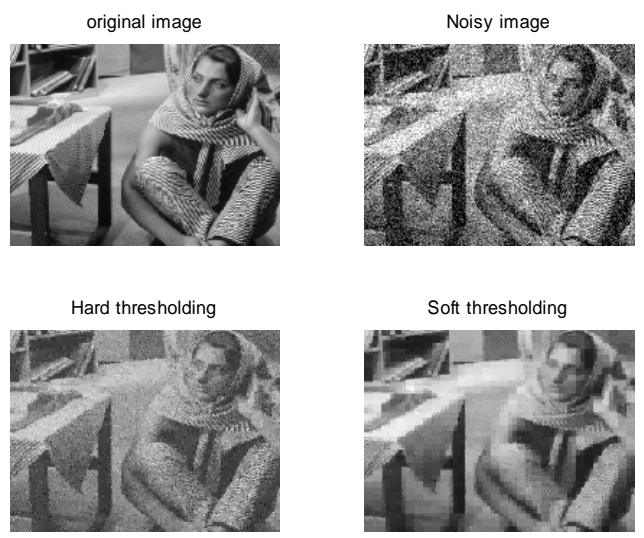

Fig. 10.For noise level $\boldsymbol{\sigma}=15$ for db1 using Visu shrink method for 4 level of decomposition
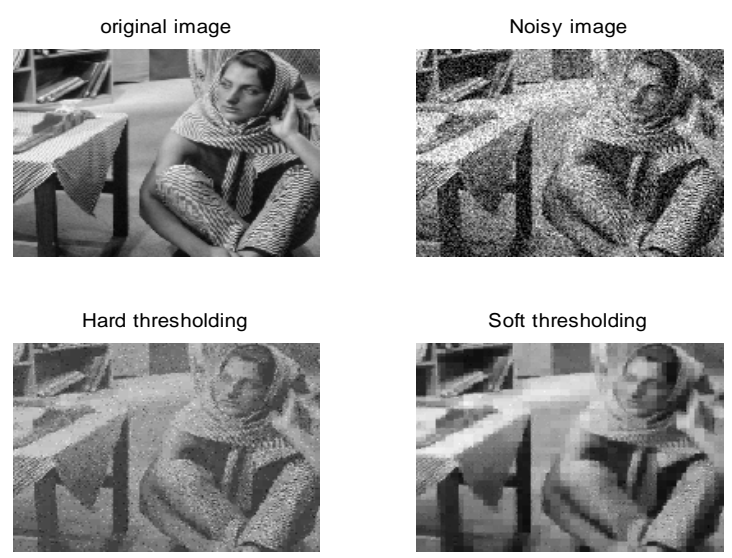

Fig. 11.For noise level $\boldsymbol{\sigma}=15$ for db1 using proposed method for 4 level of decomposition 
VI.

\section{CONCLUSION}

In this paper we have proposed the new method which is obtained by modifying the traditional Visu shrink thresholding techniques for image denoising based on wavelet transform. The result is obtained by using the Matlab software on standard Barbara image of size $512 * 512$ for Gaussian noise of noise level 0.02 . The main drawback of Visu shrink techniques is that it produce over smoothen image which is caused by increase in decomposition level which in turn increase threshold value and hence increase smoothening of image. In our proposed method we maintain the threshold value hence smoothening effect is reduce. We have compared the Peak signal to noise ratio (PSNR) value of Visu Hard thresholding Method with our proposed method for different wavelet families such as Haar, Db1, Bior1.5, Sym1 and Coif1. It was seen that with different Wavelet Families our proposed method gave better PSNR value. Our method also produces better result for other noises such as Salt and pepper, Speckle and Poisson.

\section{REFERENCES}

[1] Ming Tian, Hao Wen, Long Zhou and Xinge You "Image denoising using multi-scale thresholds method in the wavelet domain", Proceedings of the 2010 International conference on wavelet Analysis and Pattern Recognition, Qingdao, 11-14 July 2010.

[2] Rajesh Kumar Rai, Jyoti Asnani and T.R.Sontakke "Review of shrinkage Techniques for image denoising", International Journal of Computer Applications, (0975-8887) Volume42 -No.19, March 2012.

[3] Rakesh Kumar and B.S.Saini "Improved Image denoising Technique Using Neighbouring Wavelet Coefficients of optimal wavelet with adaptive thresholding", International Journal of Computer Theory and Engineering, Vol.4, No.3, June 2012.

[4] Hari Om and Mantosh Biswas “A generalized image denoising method using neighbouring Wavelet coefficients", Springer Verlag London 2013.

[5] Hari Om and Mantosh Biswas "Improved Image Denoising Method Based on Wavelet Thresholding", Journal of signal and information processing,2012, 3, 109-116.

[6] Imam Elyasi and Sadegh Zarmehi, "Elimination of Noise By Adaptive Wavelet threshold", World Academy of Science", Engineering and Technology, 562009.

[7] M. Neelima and Md. Mahaboob Pasha, "Wavelet Transform Based on Image Denoising Using Thresholding Techniques", International Journal of Advanced Research in computer and communication Engineering, Vol.3, Issue 9, September 2014 ISSN (online): 2278-1021, ISSN (print): 2319-5940.

[8] Sachin D Ruikar and Dharmpal D Doye "Wavelet based Image denoising Techniques", (IJACSA) International Journal of Advanced Computer Science and Applications, Vol.2, No.3, March 2011.

[9] S.Kalavathy and R.M.Suresh "Anaysis of Image Denoising using Wavelet Coefficient and adaptive subband Thresholding techniques", IJCSI International Journal of computer science, Issues, Vol. 8, Issue6, No.1, November 2011 ISSN (online): 1694-0814 www.IJCSI.org.

[10] Fatma H. Elfouly, Mohamed I. Mahmoud, Moawad I. M. Dessouky and Salah Deyab “ Comparison Between Haar and Daubechies Wavelet Transformations on FPGA Technology", International Journal of Computer, Information, and Systems Science, and Engineering, 2:1 (C) www.waset.org Winter 2008

[11] Dipalee Gupta and Siddartha Choubey, "Discrete Wavelet Transform for Image Processing”, International Journal of Emerging Technology and Advanced Engineering, Website: www.ijetae.com (ISSN 2250-2459, ISO 9001: 2008 certified Journal, Volumes 4, Issue 3, March 2015.

[12] Lakshmi Bhamidipati, Sai Sudha Mindagudla, Harsha Vardhan Devlla, Hima Sagar Goodi and Hemanth Nag, "Analysis of Different Discrete Wavelet Transform Basis Functions in Speech Compression", IOSR Journal of VLSI and Signal Processing(IOSR-JVSP) volume 4, Ver.II (Jan.2014), PP 34-38 e-ISSN: 2319- 4200, p-ISSN No. : 2319 - 4197 www.iosrjournals.org.

[13] Dikendra Verma and Sanjay Mathur “ Image denoising Based on Averaging Of Two Wavelet Transformed Images”, International Journal of Engineering Research and Applications (IJERA), ISSN: 2248-9622www.ijera.com Vol. 2, Issue 5, September - October 2012, pp 2112 2119

\section{AUTHORS PROFILE}

Pushpa Koranga: She is currently pursuing M.Tech from Electronics and Communication specialization in Communication System from Graphic Era Hill University, Bhimtal Uttarakhand, India. She has received Bachelor degree from Electronics and Communication Engineering with honors from Uttaranchal Institute of Technology (UIT), Dehradoon, India in 2015. Her area of interest includes Image processing

Garima Singh: She is currently pursuing M.Tech from Electronics and Communication specialization in Communication System from Graphic Era Hill University, Bhimtal, Uttarakhand, India. She has received Bachelor degree from Electronics and communication engineering from Soorajmal Laxmidevi Sawarthia Educational Trust Group of Institution, Kichha U.S nagar, India in 2015. Her area of interest includes Image processing.

Dikendra Verma: He is currently Assistant Professor in Graphic Era Hill University, Bhimtal Uttarakhand, India. He has received Bachelor degree from Dehradoon Institute of Technology (DIT) Dehradoon, India in 2006. He also received his Master's Degree in Electronics and Communication with Major in Electronics and Minor in Computer Science from G.B Pant University of Agriculture and Technology, Pantnagar, Uttarakhand, India in 2012. His area of interest includes Image processing. 